\title{
Significance of lung biopsy for the definitive diagnosis of lung nodules in breast cancer patients
}

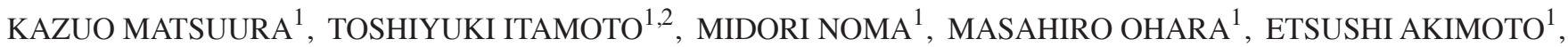 \\ MIHOKO DOI $^{3}$, TAKASHI NISHISAKA ${ }^{4}$, KOJI ARIHIRO $^{5}$, TAKAYUKI KADOYA $^{6}$ and MORIHITO OKADA ${ }^{6}$ \\ ${ }^{1}$ Department of Breast Surgery, Hiroshima Prefectural Hospital, Hiroshima, Hiroshima 734-8530; ${ }^{2}$ Faculty of Medicine, \\ Hiroshima University, Hiroshima, Hiroshima 734-8553; Departments of ${ }^{3}$ Clinical Oncology and ${ }^{4}$ Clinical Laboratory, \\ Hiroshima Prefectural Hospital, Hiroshima, Hiroshima 734-8530; Departments of ${ }^{5}$ Pathology and ${ }^{6}$ Surgical Oncology, \\ Hiroshima University Hospital, Hiroshima, Hiroshima 734-8551, Japan
}

Received August 31, 2017; Accepted October 18, 2017

DOI: $10.3892 / \mathrm{mco} .2017 .1511$

\begin{abstract}
The aim of the present study was to evaluate the significance of lung biopsy for the modification of the treatment strategy in breast cancer patients who develop lung nodules during follow-up after breast surgery. Of 53 consecutive patients who underwent lung biopsies in two institutions (Hiroshima University Hospital and Hiroshima Prefectural Hospital, Hiroshima, Japan) between 1997 and 2014, 45 underwent lung surgery and 8 underwent percutaneous or transbronchial tumor biopsy for lung nodules developing after curative surgery for breast cancer. The indications for lung biopsy included lung nodules for which a definitive diagnosis was difficult to achieve, and those for which the treatment strategy depended on the pathological diagnosis. The lung nodules were pathologically diagnosed as primary breast cancer metastases to the lungs in $25(47 \%)$, primary malignant lung tumors in $21(40 \%)$ and benign disease in 7 (13\%) patients. Among the 25 metastatic patients confirmed by lung biopsy, phenotype discordance was observed in 6 patients (24\%). A total of 3 patients with lung metastasis proven to have estrogen or progesterone receptor upregulation by lung biopsy received endocrine therapy. Univariate analysis revealed that patients with metastatic breast cancer confirmed by lung biopsy were significantly younger and had more locally advanced primary cancers diagnosed via clinical and pathological assessment compared with patients with other diseases. Therefore, mastectomy and axillary lymph node dissection were performed more frequently in the metastasis group compared with the others group. Multivariate analysis revealed that mastectomy $(\mathrm{P}<0.001)$ and axillary dissection $(\mathrm{P}<0.001)$ were independent factors predicting that the lung nodules would be metastases
\end{abstract}

Correspondence to: $\mathrm{Dr}$ Kazuo Matsuura, Department of Breast Surgery, Hiroshima Prefectural Hospital, 1 Chome-5-54, Minami-ku, Ujinakanda, Hiroshima, Hiroshima 734-8530, Japan E-mail:m79kazuo@gmail.com

Key words: breast cancer, lung nodule, lung metastases from breast cancer. Lung biopsy in breast cancer patients who developed lung nodules during the follow-up period after breast cancer surgery was crucial for making a definitive diagnosis and modifying the treatment strategy, which may improve the prognosis of breast cancer patients.

\section{Introduction}

Breast cancer has been confirmed by gene expression analysis to be a heterogeneous disease $(1,2)$ that may be divided into biologically distinct subtypes. In clinical practice, the cancer subtype is determined using immunohistochemistry $(3,4)$ and a therapeutic plan is designed according to the subtype (5). Lung metastasis is commonly observed in breast cancer patients at the time of relapse. Patients with metastatic disease have median survival times of 12-24 months $(6,7)$. The majority of patients with breast cancer metastases to the lungs are treated with systemic therapy. However, lung nodules that develop in breast cancer patients during follow-up after curative breast surgery may not always represent metastatic lesions. Therefore, the final treatment strategy for breast cancer patients with lung nodules depends on the pathological diagnosis of the nodules. However, it is difficult to obtain an accurate diagnosis only by using imaging modalities, such as computed tomography (CT), particularly in patients with solitary nodules. For a definitive diagnosis of the lung lesions, tumor biopsy, such as transbronchial or CT-guided biopsy, may be performed. Video-assisted thoracoscopic surgery (VATS) with intraoperative inspection is an optimal procedure for the complete removal of the lesions and for reaching a definitive diagnosis, but the survival benefit of total biopsy of lung nodules by VATS remains to be established.

The aim of the present study was to evaluate the significance of lung biopsy, including total biopsy by VATS, in breast cancer patients who develop lung nodules during follow-up after curative breast surgery.

\section{Patients and methods}

Patients. In total, 53 consecutive patients who underwent lung biopsy following curative surgery for breast cancer in two 
institutions (Hiroshima University Hospital and Hiroshima Prefectural Hospital, Hiroshima, Japan) between 1995 and 2014 were enrolled in this retrospective study. The age at lung biopsy was 27-84 years (mean, 63 years). A total of 30 patients (57\%) had a solitary lung nodule, 9 (17\%) had 2 nodules, and 14 (26\%) had $\geq 3$ nodules. VATS was performed in 45 patients, transbronchial lung biopsy in 7 patients, and CT-guided biopsy in 1 patient. The indications for lung biopsy included lung nodules that were difficult to diagnose clinically, and those for which the treatment strategy would depend on the pathological diagnosis.

In the event of malignant lung tumors, a non-curative lung biopsy was defined as a macroscopically visible incomplete resection. When lung biopsy was performed in patients with metastatic disease at extrapulmonary sites, the procedure was also non-curative.

The majority of the patients who were included in the present study underwent curative surgery for primary breast cancer at one of the two aforementioned institutions and they were subsequently followed up. The clinical records and pathological reports of patients who underwent surgery for primary breast cancer at other hospitals and presented to our hospital with lung nodules were obtained from the respective hospitals. Use of these data was approved by the Institutional Review Board (H27-073).

Patients were divided into the metastasis (patients with metastatic lung cancer from breast cancer; $n=25$ ) and others (patients with other pathologies; $\mathrm{n}=28$ ) groups. In the present study, we focused on the survival benefits of lung biopsy in the metastasis group compared with the others group with variable backgrounds, as the number of patients with primary lung cancer or benign lung tumors was limited. Various clinicopathological factors recorded at the time of primary breast cancer surgery were compared between the groups, and a subgroup analysis was performed in the metastasis group to compare their outcomes after lung biopsy.

Histological assessment. Routine hematoxylin and eosin staining was performed on sections from tumor specimens in order to determine the histological tumor type. Nuclear grade was determined according to the 17th edition of the general rules for clinical and pathological recording of breast cancer of the Japanese Breast Cancer Society (8). Immunohistochemical staining was performed to evaluate the expression status of estrogen receptor (ER), progesterone receptor (PR), human epidermal growth factor receptor 2 (HER2) and $\mathrm{Ki}-67$, as previously described (9). The following monoclonal antibodies were used in the analysis: ER (SP1; 790-4324, prediluted, Ventana Medical Systems, Tucson, AZ, USA); PR (1E2; 790-2223, prediluted, Ventana Medical Systems); Ki-67 (MIB-1; M7240, 1:80 dilution, DAKO, Glostrup, Denmark); and HER2 (4B5; 790-2991, prediluted, Ventana Medical Systems). Based on the expression of ER, PR, HER 2 and $\mathrm{Ki}-67$, patients were classified as having one of the following subtypes, as previously described (9): Luminal A, luminal B HER2-negative, luminal B HER2-positive, HER2-positive and triple-negative types.

Statistical analysis. Data are presented as number (\%) or as mean, unless otherwise stated. Categorical variables in both groups were compared using Pearson's Chi-squared test, and small samples were assessed using Fisher's exact test.
The overall survival (OS) in the metastasis group was calculated from the date of lung biopsy to the date of death from any cause, or the date of the last follow-up. The Kaplan-Meier method was used to calculate OS, and patient subgroups were compared using the log-rank test. All statistical analyses were performed using EZR (Saitama Medical Center, Jichi Medical University: http://www.jichi.ac.jp/saitama-sct/SaitamaHP. files/statmedEN.html; Kanda, 2012), which is a graphical user interface for R statistical software, version 2.13.0 (The R Foundation for Statistical Computing, Vienna, Austria). More precisely, it is a modified version of the $\mathrm{R}$ commander (version 1.6-3) that was designed to include statistical functions frequently used in biostatistics (10).

\section{Results}

Characteristics of primary breast cancer patients with lung nodules. All patients who underwent lung biopsy had no major fatal complications, although there were a few minor complications, such as air leakage caused by the biopsy of peripheral lung nodules. The pathological diagnoses of the lung nodules included breast cancer metastases to the lungs in 25 (47\%), primary malignant lung tumor in $21(40 \%)$ and benign disease in 7 patients (13\%). Of the 21 patients with primary malignant lung tumors, 17 had adenocarcinoma, 2 had large-cell carcinoma, 1 had small-cell carcinoma and 1 had a carcinoid tumor. Of the 7 patients with benign disease, 4 had inflammation, 2 had organizing pneumonia and 1 had a hamartoma. Of the 25 patients with breast cancer metastases to the lungs, curative lung biopsy was performed in 14 patients (56\%). Of the 21 patients with primary malignant lung tumors, $18(86 \%)$ had stage I disease and 19 (90\%) underwent curative lung biopsy (Fig. 1).

The comparison of the clinical factors between the metastasis and the others groups at the time of primary breast surgery by univariate analysis revealed that the patients in the metastasis group were significantly younger compared with those in the others group (median age, 59 vs. 65 years, respectively; $\mathrm{P}<0.001)$. The rate of premenopausal status was significantly higher in the metastasis group compared with that in the others group (56 vs. $14 \%$, respectively; $\mathrm{P}=0.003$ ). The patients in the metastasis group had higher node-positive rates compared with those in the others group (56 vs. $11 \%$, respectively; $\mathrm{P}=0.001)$. The clinical stage of the patients in the metastasis group was also higher compared with that in the others group $(\mathrm{P}=0.027)$. Therefore, mastectomy (64 vs. $18 \%$, respectively; $\mathrm{P}<0.001$ ) and axillary lymph node dissection (96 vs. $32 \%$, respectively; $\mathrm{P}<0.001$ ) were performed more frequently in the metastasis group compared with the others group. Postoperative radiotherapy and primary systemic chemotherapy were administered more frequently in the metastasis group compared with the others group (56 vs. 18\%, $\mathrm{P}=0.005$ and 80 vs. $32 \%, \mathrm{P}<0.001$, respectively;). The clinicopathological characteristics of the patients are summarized in Tables I and II.

The comparison of the pathological factors between the groups at the time of primary breast surgery by univariate analyses revealed that the patients in the metastasis group had higher node-positive rates compared with those in the others group (79 vs. 52\%, respectively; $\mathrm{P}=0.0492$ ). The rate of 


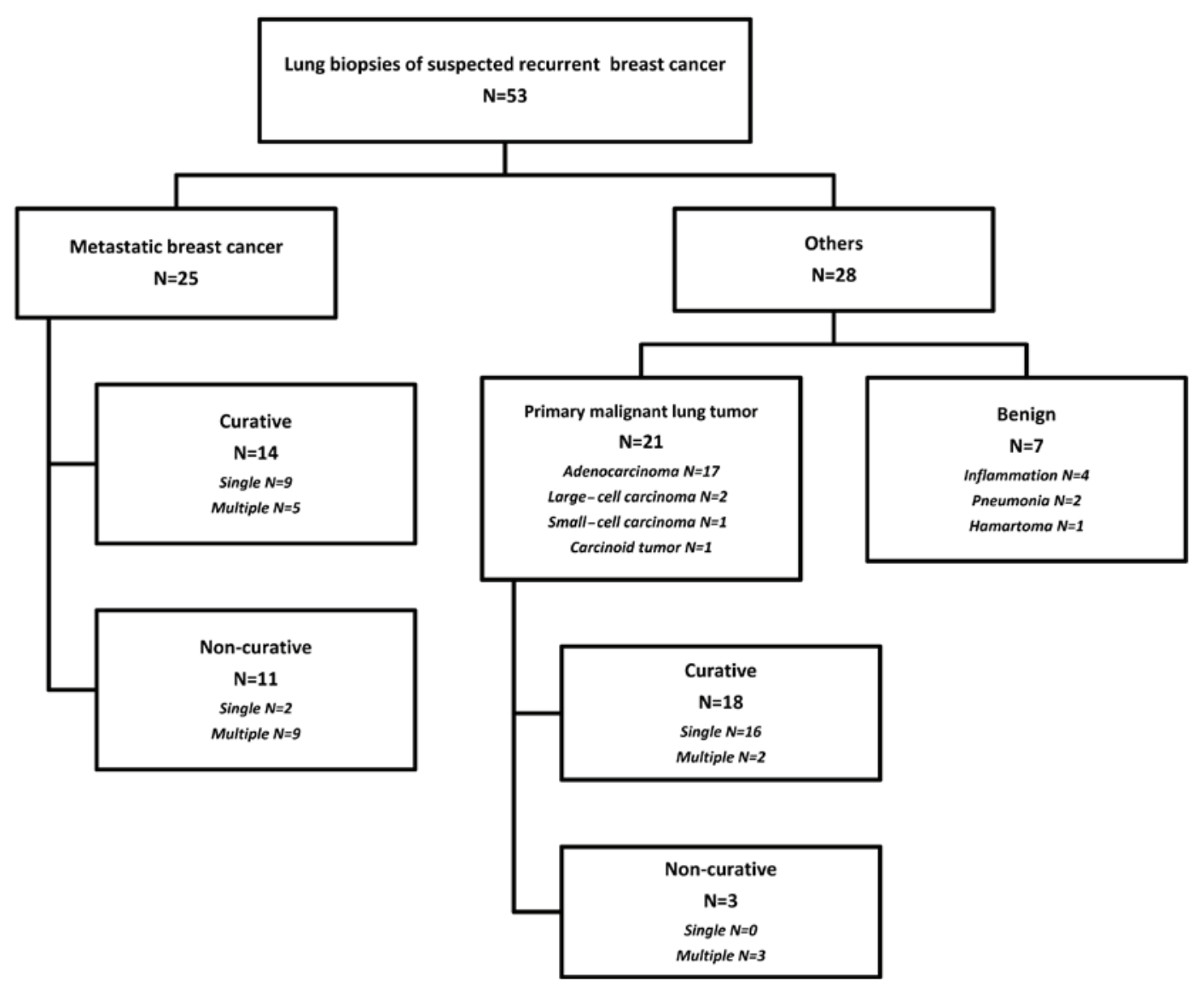

Figure 1. Histological diagnosis of lung nodules in breast cancer patients.

positive lymphovascular invasion was significantly higher in the metastasis group compared with that in the others group (52 vs. $25 \%$, respectively; $\mathrm{P}=0.004$ ).

The mean disease-free interval from the surgery for primary breast cancer to lung biopsy, multiplicity of lung nodules, and curability by lung biopsy did not differ significantly between the two groups (Table III).

Multivariate analysis revealed that mastectomy $(\mathrm{P}<0.001)$ and axillary resection $(\mathrm{P}<0.001)$ were independent factors predicting whether the lung nodules would be metastases from breast cancer (Table I).

Survival analysis. In the metastasis group, 11 patients with solitary nodules had significantly better survival rates compared with 14 patients with multiple nodules (3-year survival rates, 100 vs. $74.3 \%$, respectively; $\mathrm{P}=0.00104$; Fig. 2). Moreover, 14 patients who underwent curative lung biopsy had significantly higher survival rates compared with 11 patients who underwent non-curative biopsy (3-year survival rates, 89 vs. $81 \%$, respectively; $\mathrm{P}=0.00673$; Fig. 3 ).

Discordance in subtype between the primary tumor and metastases. A total of 17 patients with metastases maintained the same tumor phenotype as the primary tumor, whereas discordance of the ER, PR, HER2, or Ki-67 expression status was observed between the primary and metastatic tumors in 6 patients $(24 \%)$. A total of 3 patients with ER or PR upregulation received endocrine therapy instead of chemotherapy following identification of discordance (Table IV).

\section{Discussion}

Although lung nodules may develop during the follow-up period in breast cancer patients, they are not necessarily metastases from the primary breast cancer but may correspond to other pathologies, such as primary lung cancer or benign lesions. The choice of treatment strategy largely depends on the pathology. In the event of pathologically confirmed lung metastasis, the phenotype of the tumor may be identified from the analysis of the specimens, in order to determine whether the phenotype of the metastatic lesion is in concordance or discordance with that of the primary lesion. If discordance is detected, the systemic therapy regimen must be selected accordingly. Thus, based on the advantages of a definitive pathological diagnosis for recurrent breast cancer, the international guidelines for breast cancer recommend a biopsy for recurrent lesions whenever possible (11).

Previous studies have demonstrated that patients with breast cancer are at high risk of developing secondary malignancies (12-15). In the present study, over half of the lung nodules that developed during the follow-up period after breast cancer surgery were not lung metastases from breast cancer, but comprised a variety of histological diagnoses, including 21 primary lung cancers. Jensen et al also reported that the biopsies from the suspicious metastatic lesions revealed benign disease or other malignancies in $14 \%$ of the patients (16), whereas Tanaka et al (17) demonstrated that $75 \%$ of the 52 patients who underwent surgery for lung nodules that developed during the follow-up period after breast cancer 
Table I. Clinical characteristics of primary breast cancer.

\begin{tabular}{|c|c|c|c|c|}
\hline Variables & Metastasis $(\mathrm{n}=25)$ & Others $(n=28)$ & P-value & Multivariate P-value \\
\hline Median age, years (range) & $59(25-63)$ & $65(41-78)$ & $<0.001$ & \\
\hline Menopausal status & & & 0.003 & - \\
\hline Premenopausal/postmenopausal & $14 / 11$ & $4 / 24$ & & \\
\hline BMI, kg/m² (range) & $22.4(16.6-30.6)$ & $23.6(17.4-34.2)$ & 0.232 & \\
\hline Patients with other cancers & & & 0.183 & \\
\hline Yes/no & $22 / 3^{b}$ & $20 / 8^{c}$ & & \\
\hline Contralateral breast cancer & & & 0.113 & \\
\hline Yes/no & $0 / 25$ & $4 / 24$ & & \\
\hline Breast/ovarian cancer family history & & & 0.404 & \\
\hline Yes/no & $4 / 21$ & $2 / 26$ & & \\
\hline Clinical tumor stage & & & 0.417 & \\
\hline Tis/T1,2/T3,4 & $0 / 22 / 3$ & $3 / 23 / 2$ & & \\
\hline Clinical node stage & & & 0.001 & - \\
\hline Negative/positive & $11 / 14$ & $25 / 3$ & & \\
\hline Clinical stage & & & 0.027 & \\
\hline 0/I/II/III & $0 / 8 / 12 / 5$ & $3 / 16 / 8 / 1$ & & \\
\hline Type of breast surgery & & & $<0.001$ & 0.045 \\
\hline Mastectomy/partial mastectomy & $16 / 9$ & $5 / 23$ & & \\
\hline Axillary LN dissection & & & $<0.001$ & 0.001 \\
\hline None or SLNB/Ax & $1 / 24$ & $19 / 9$ & & \\
\hline Radiation therapy & & & 0.005 & - \\
\hline Yes/no & $11 / 14$ & $23 / 5$ & & \\
\hline Chemotherapy ${ }^{\mathrm{a}}$ & & & $<0.001$ & - \\
\hline Yes/no/unknown & $20 / 4 / 1$ & $9 / 19 / 0$ & & \\
\hline Hormonal therapy & & & 0.321 & \\
\hline Yes/no/unknown & $13 / 11 / 1$ & $19 / 9 / 0$ & & \\
\hline
\end{tabular}

${ }^{\mathrm{a}}$ Chemotherapy includes primary systemic chemotherapy. ${ }^{\mathrm{b}}$ Thyroid, lung, stomach. ${ }^{\mathrm{c}}$ Ovary, esophagus, rectum, oral, pancreas, brain, duodenum, except for secondary lung cancer. BMI, body mass index; metastasis, breast cancer metastases; LN, lymph node; SLNB, sentinel lymph node biopsy; Ax, axillary lymph node dissection. The patient population was subdivided according to histological diagnosis of breast cancer metastases or others.

surgery had pathologically confirmed metastases from the primary breast cancer. In the present study, of the 53 patients with lung nodules, $47 \%$ of those who underwent lung biopsy had metastasis from the primary breast cancer. However, this proportion was lower compared with that reported in previous studies $(16,17)$. This may be attributed to the fact that the patients included in our cohort were highly selected, i.e., the indications for lung biopsy were restricted to only lung nodules with difficult clinical diagnoses. When the lung nodules were proven to be metastatic based on the clinical course and imaging findings, it was decided that there was no indication for lung biopsy, although this policy was not in accordance with the recommendations of the international guidelines published in 2015 (11).

Rena et al (18) demonstrated that there were no statistically significant differences between the radiological characteristics of lung nodules and the pathological profiles of the metastases from primary breast cancer, primary lung cancer, or benign tumors. The present study demonstrated that the patients in the metastasis group were younger compared with those in the others group, and the clinicopathological characteristics of the primary cancer in the metastasis group indicated more advanced disease compared with the others group. However, a definitive diagnosis of the lung nodules that develop during the follow-up period as metastases from the primary breast cancer is difficult to make based on the clinicopathological characteristics of the primary cancer alone.

The lack of evidence regarding any survival benefits conferred by surgical treatment of breast cancer metastases to the lung renders the procedure controversial. Fan et al (19) demonstrated in a meta-analysis that the pooled 5-year survival rate after lung metastasectomy in breast cancer patients was $46 \%$. They also demonstrated the prognostic value of complete resection of metastases and solitary lung metastasis. The present study investigated the outcomes in patients with solitary metastases when surgical resection was performed as a total biopsy of the lung nodule. The survival benefits of metastasectomy should be further discussed, as there are no 
Table II. Pathological characteristics of primary breast cancer.

\begin{tabular}{|c|c|c|c|c|}
\hline Variables & Metastasis $(n=25)$ & Others $(n=28)$ & P-value & Multivariate P-value \\
\hline Pathological tumor stage; (y)pT & & & 0.229 & \\
\hline Tis/T1,2/T3,4 & $0 / 21 / 4$ & $4 / 23 / 1$ & & \\
\hline Pathological node stage; (y)pN & & & 0.0492 & - \\
\hline Negative/positive & $13 / 12$ & $22 / 6$ & & \\
\hline Pathological stage & & & 0.101 & \\
\hline 0/I/II/III & $0 / 8 / 10 / 7$ & $4 / 13 / 7 / 4$ & & \\
\hline Lymphovascular invasion & & & 0.004 & - \\
\hline Negative/positive/unknown & $4 / 13 / 8$ & $17 / 7 / 4$ & & \\
\hline Nuclear grade & & & 0.0552 & \\
\hline 1/2/3/unknown & $3 / 3 / 8 / 11$ & $6 / 11 / 4 / 7$ & & \\
\hline \multicolumn{5}{|l|}{ ER status } \\
\hline Negative/positive/unknown & $8 / 15 / 2$ & $5 / 23 / 0$ & 0.207 & \\
\hline PR status & & & 0.167 & \\
\hline Negative/positive/unknown & $12 / 11 / 2$ & $9 / 19 / 0$ & & \\
\hline HER2 status, n (\%) & & & 0.092 & \\
\hline $0 / 1+/ 2+/ 3+/$ unknown & $7 / 4 / 5 / 4 / 5$ & $5 / 11 / 3 / 1 / 7$ & & \\
\hline \multicolumn{5}{|l|}{$\mathrm{Ki}-67$} \\
\hline$<20 \% />20 \% /$ unknown & $7 / 11 / 7$ & $7 / 6 / 15$ & 0.481 & \\
\hline Tumor subtype, n (\%) & & & 0.168 & \\
\hline Luminal/non-luminal & $16 / 7$ & $21 / 3$ & & \\
\hline
\end{tabular}

ER, estrogen receptor; PR, progesterone receptor; HER2, human epidermal growth factor receptor 2.

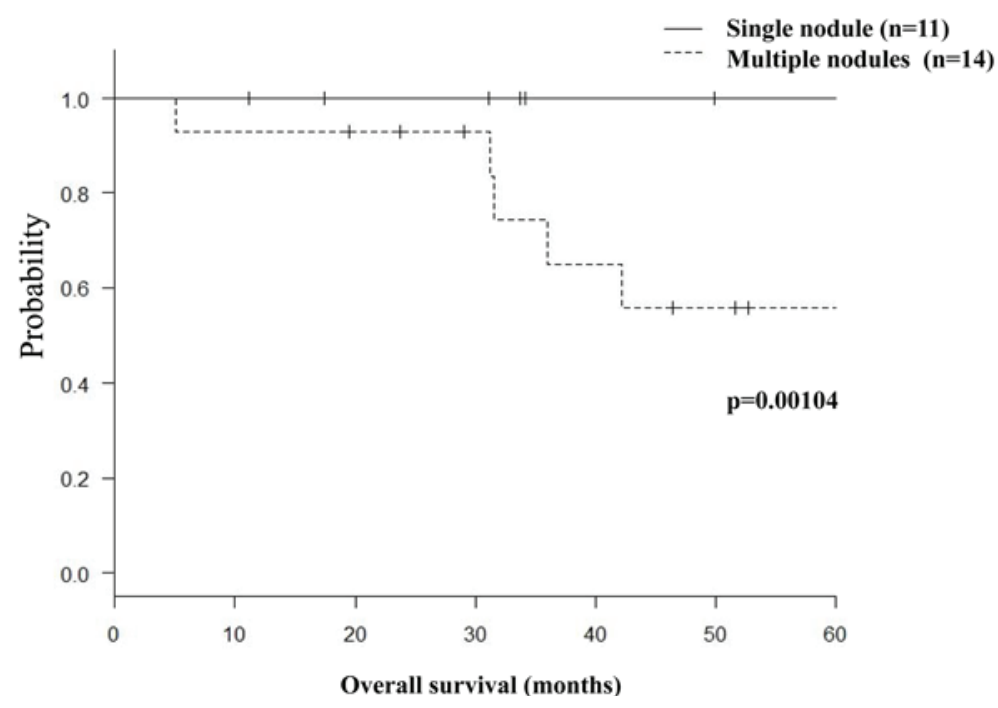

Figure 2. Overall survival according to the number of lung metastases.

long-term data available and the number of cases in the present retrospective study was limited, with variable backgrounds.

Changes in the HER2 and hormone receptor status of metastatic foci from primary cancer may lead to the modification of treatment strategies based on the indications for HER2-targeted or endocrine therapies. Previous studies reported discordance rates of $10-40 \%$ for the hormone receptor status and 5-20\% for the HER2 status (20-22). In the present study, the discordance rates for hormone receptor and HER 2 status were 12 and 8\%, respectively. Welter et al (23) demonstrated that the number of metastases, tumor stage at initial presentation, complete resection, and pleural/chest wall involvement were not prognostic factors of survival. Instead, ER expression predicted prolonged survival, with a 5-year survival rate of $76 \%$ for ER-positive and $12 \%$ for ER-negative tumors, whereas similar statistically significant differences 
Table III. Characteristics of patients with lung nodules.

\begin{tabular}{|c|c|c|c|c|}
\hline Variables & Metastases $(n=25)$ & Others $(n=28)$ & P-value & Multivariate P-value \\
\hline Disease-free interval, months & & & 0.325 & \\
\hline Mean (SD) & $66.3(45.5)$ & $52.7(53.8)$ & & \\
\hline No. of lung nodules & & & 0.101 & - \\
\hline Solitary/multiple & $11 / 14$ & $19 / 9$ & & \\
\hline \multicolumn{5}{|l|}{ Resection of metastases } \\
\hline Curative/non-curative & $14 / 11$ & $22 / 6$ & 0.139 & - \\
\hline
\end{tabular}

$\mathrm{SD}$, standard deviation.

Table IV. Discordance in subtype between the primary tumor and metastases.

\begin{tabular}{llll}
\hline Variables & & $\mathrm{n}=25(\%)$ & Patient status (n) \\
\hline Concordant phenotype & & $17(68)$ & $\begin{array}{l}\text { Alive (7) } \\
\text { Deceased (10) } \\
\text { Discordant phenotype }\end{array}$ \\
& Change of HR, HER2 and Ki-67 & $6(24)$ & Alive (4) \\
Luminal A $\rightarrow$ luminal B & Upregulation of Ki-67 & $1(17)$ & Deceased (2) \\
Luminal HER2 $\rightarrow$ luminal B & Loss of HER2 & $2(33)$ & Alive \\
Triple-negative $\rightarrow$ luminal B & Upregulation of ER and/or PR & $3(50)$ & Deceased \\
N/A phenotype & & $2(8)$ & Alive \\
& & & Alive (1) \\
& & Deceased (1)
\end{tabular}

HR, hormone receptor; ER, estrogen receptor; PR, progesterone receptor; HER2, human epidermal growth factor receptor 2; N/A, not available.

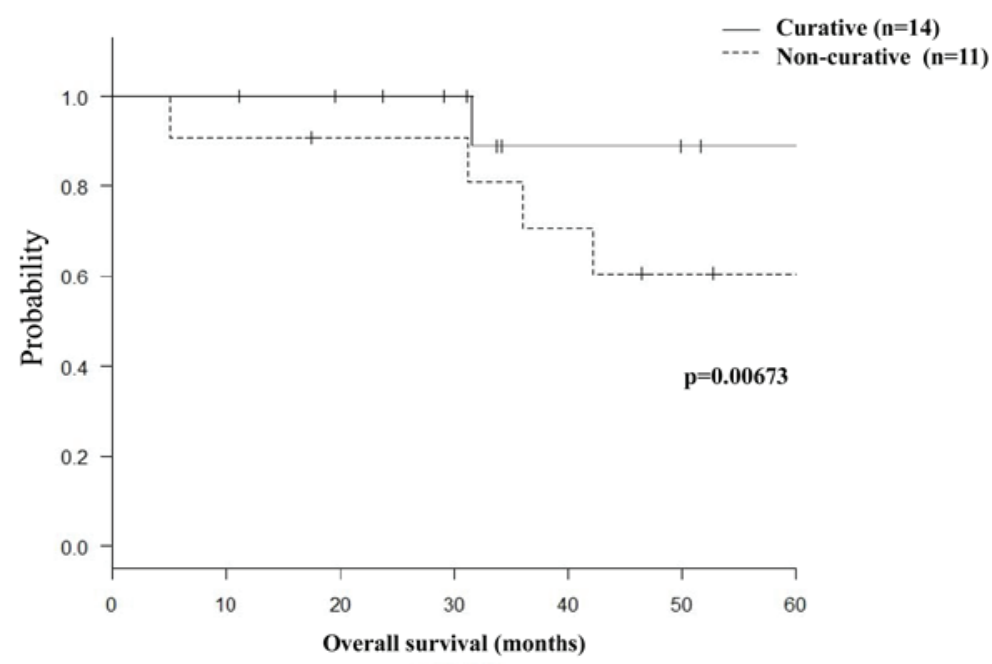

Figure 3. Overall survival according to the resectability of lung metastases.

were identified according to the HER2 expression status (23). The appropriate introduction of endocrine therapies may contribute to longer survival in the future.

Patients with other lung pathologies identified by biopsy may also benefit from lung biopsy. The majority of patients with primary malignant lung tumors confirmed by biopsy had early-stage disease and were able to undergo curative resection.
This allows the circumvention of unnecessary systemic therapies for metastatic breast cancer. Patients with benign pathologies confirmed by biopsy may also benefit from lung biopsy.

In conclusion, the clinical diagnosis of lung nodules that developed during follow-up after curative breast surgery remains difficult, despite the current developments in imaging techniques. Although the clinicopathological profiles of 
primary breast cancers provide useful information regarding the differential diagnosis of lung nodules, lung biopsy is key to reaching a definitive diagnosis and designing the subsequent treatment strategy. Moreover, it may improve the prognosis when metastatic lung nodules from primary breast cancer are resected with curative intent.

\section{References}

1. Sørlie T, Perou CM, Tibshirani R, Aas T, Geisler S, Johnsen H, Hastie T, Eisen MB, van de Rijn M, Jeffrey SS, et al: Gene expression patterns of breast carcinomas distinguish tumor subclasses with clinical implications. Proc Natl Acad Sci USA 98: 10869-10874, 2001.

2. Perou CM, Sørlie T, Eisen MB, van de Rijn M, Jeffrey SS, Rees CA, Pollack JR, Ross DT, Johnsen H, Akslen LA, et al: Molecular portraits of human breast tumours. Nature 406: 747-752, 2000.

3. Cheang MC, Chia SK, Voduc D, Gao D, Leung S, Snider J, Watson M, Davies S, Bernard PS, Parker JS, et al: Ki67 index, HER2 status and prognosis of patients with luminal B breast cancer. J Natl Cancer Inst 101: 736-750, 2009.

4. Prat A, Cheang MC, Martín M,Parker JS, Carrasco E, Caballero R, Tyldesley S, Gelmon K, Bernard PS, Nielsen TO and Perou CM: Prognostic significance of progesterone receptor-positive tumor cells within immunohistochemically defined luminal A breast cancer. J Clin Oncol 31: 203-209, 2013.

5. Coates AS, Winer EP, Goldhirsch A, Gelber RD, Gnant M, Piccart-Gebhart M, Thürlimann B and Senn HJ; Panel Members: Tailoring therapies-improving the management of early breast cancer: St Gallen International Expert Consensus on the Primary Therapy of Early Breast Cancer 2015. Ann Oncol 25: 1533-1546, 2015.

6. Rashid OM and Takabe K: The evolution of the role of surgery in the management of breast cancer lung metastasis. J Thorac Dis 4: 420-424, 2012.

7. Siegel RL, Miller KD and Jemal A: Cancer statistics, 2015. CA Cancer J Clin 65: 5-29, 2015.

8. Society TJBC: General rules for clinical and pathological recording of breast cancer, 2012.

9. Ohara M, Akimoto E, Noma M, Matsuura K, Doi M, Kagawa N and Itamoto T: Prognostic impact of progesterone receptor status combined with body mass index in breast cancer patients treated with adjuvant aromatase inhibitor. Oncol Lett 10: 3286-3292, 2015

10. Kanda Y: Investigation of the freely available easy-to-use software 'EZR' for medical statistics. Bone Marrow Transplant 48: 452-458, 2013.

11. Gradishar WJ, Anderson BO, Balassanian R, Blair SL, Burstein HJ, Cyr A, Elias AD, Farrar WB, Forero A, Giordano SH, et al: Breast Cancer Version 2.2015. J Natl Compr Canc Netw 13: 448-475, 2015.

12. Brown LM, Chen BE, Pfeiffer RM, Schairer C, Hall P, Storm H, Pukkala E, Langmark F, Kaijser M, Andersson M, et al: Risk of second non-hematological malignancies among 376,825 breast cancer survivors. Breast Cancer Res Treat 106: 439-451, 2007.
13. Kirova YM, De Rycke Y, Gambotti L, Pierga JY, Asselain B and Fourquet A; Institut Curie Breast Cancer Study Group: Second malignancies after breast cancer: The impact of different treatment modalities. Br J Cancer 98: 870-874, 2008.

14. Shilkrut M, Belkacemi Y and Kuten A; Association of Radiotherapy and Oncology of the Mediterranean arEa (AROME): Secondary malignancies in survivors of breast cancer: How to overcome the risk. Crit Rev Oncol Hematol 84 (Suppl 1): e86-e89, 2012.

15. Vidal-Millan S, Zeichner-Gancz I, Flores-Estrada D, Vela-Rodríguez BE, Vazquez-López MI, Robles-Vidal CD, Ramirez-Ugalde MT and Chávez-MacGregor M: A descriptive study of second primary malignancies associated to breast cancer in a mexican Hispanic population. Med Oncol 22: 17-22, 2005.

16. Jensen JD, Knoop A, Ewertz M and Laenkholm AV: ER, HER2, and TOP2A expression in primary tumor, synchronous axillary nodes and asynchronous metastases in breast cancer. Breast Cancer Res Treat 132: 511-521, 2012.

17. Tanaka F, Li M, Hanaoka N, Bando T, Fukuse T, Hasegawa S and Wada H: Surgery for pulmonary nodules in breast cancer patients. Ann Thorac Surg 79: 1711-1715, 2005.

18. Rena O, Papalia E, Ruffini E, Filosso PL, Oliaro A, Maggi G and Casadio $\mathrm{C}$ : The role of surgery in the management of solitary pulmonary nodule in breast cancer patients. Eur J Surg Oncol 33: 546-550, 2007.

19. Fan J, Chen D, Du H, Shen C and Che G: Prognostic factors for resection of isolated pulmonary metastases in breast cancer patients: A systematic review and meta-analysis. J Thorac Dis 7: 1441-1451, 2015.

20. Dieci MV, Barbieri E, Piacentini F, Ficarra G, Bettelli S, Dominici M, Conte PF and Guarneri V: Discordance in receptor status between primary and recurrent breast cancer has a prognostic impact: A single-institution analysis. Ann Oncol 24: 101-108, 2013.

21. Amir E, Miller N, Geddie W, Freedman O, Kassam F, Simmons C, Oldfield M, Dranitsaris G, Tomlinson G, Laupacis A, et al: Prospective study evaluating the impact of tissue confirmation of metastatic disease in patients with breast cancer. J Clin Oncol 30: 587-592, 2012.

22. Thompson AM, Jordan LB, Quinlan P, Anderson E, Skene A, Dewar JA and Purdie CA; Breast Recurrence in Tissues Study Group: Prospective comparison of switches in biomarker status between primary and recurrent breast cancer: The breast recurrence in tissues study (BRITS). Breast Cancer Res 12: R92, 2010.

23. Welter S, Jacobs J, Krbek T, Tötsch M and Stamatis G: Pulmonary metastases of breast cancer. When is resection indicated? Eur J Cardiothorac Surg 34: 1228-1234, 2008.

This work is licensed under a Creative Commons Attribution-NonCommercial-NoDerivatives 4.0 International (CC BY-NC-ND 4.0) License. 